Research Paper

\title{
Down-regulation of miR-338-3p and Up-regulation of MACC1 Indicated Poor Prognosis of Epithelial Ovarian Cancer Patients
}

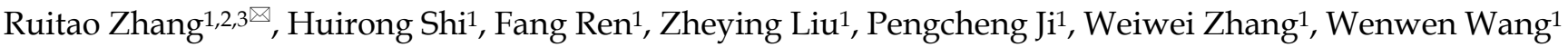 \\ 1. Department of Gynecology, First Affiliated Hospital, Zhengzhou University, Zhengzhou 450052, China \\ 2. Department of Pathology and Laboratory Medicine, University of Tennessee Health Science Center, Memphis, TN 38163, USA \\ 3. Center for Cancer Research, University of Tennessee Health Science Center, Memphis, TN 38163, USA \\ $\bowtie$ Corresponding author: Ruitao Zhang, Email: fcczhangrt@zzu.edu.cn \\ (c) Ivyspring International Publisher. This is an open access article distributed under the terms of the Creative Commons Attribution (CC BY-NC) license \\ (https://creativecommons.org/licenses/by-nc/4.0/). See http://ivyspring.com/terms for full terms and conditions.
}

Received: 2018.08.27; Accepted: 2019.01.16; Published: 2019.02.23

\begin{abstract}
Objective To detect the expression of microRNA-338-3p (miR-338-3p) and MET transcriptional regulator $\mathrm{MACCl}(\mathrm{MACCl})$ gene in different ovarian tissues, to analyze their relationships, their correlations to the clinicopathologic characteristics of epithelial ovarian cancer and their significant to the progression of ovarian cancer.

Methods The expression of miR-338-3p and MACCl gene in 20 specimens of normal ovarian tissues, 20 specimens of benign epithelial ovarian tumor and 65 specimens of epithelial ovarian cancer was detected by real-time PCR method. Their interrelationships and their correlations to the clinicopathologic characteristics of epithelial ovarian cancer were analyzed. Risk factors of recurrence and death were discussed by binary Logistic regression analysis. The relations between miR-338-3p and $\mathrm{MACCl}$ expression and the survival of ovarian cancer were measured by Kaplan-Meier analysis.

Results The expressions of miR-338-3p and $\mathrm{MACCl}$ gene in epithelial ovarian cancer tissues were $(0.331 \pm 0.038)$ and $(0.774 \pm 0.025)$, significant differences were noted between epithelial ovarian cancer and normal ovarian tissues, benign ovarian tumors $(F=77.916, P=1.205 \mathrm{E}-18 ; \mathrm{F}=77.945$, $P=1.187 \mathrm{E}-18)$. In different ovarian tissues, miR-338-3p expression was negatively correlated to MACCl expression $(r=-0.968, P<0.0001)$. In epithelial ovarian cancer, lower expression of miR-338-3p and higher expression of MACCl were associated with more advanced FIGO stage, higher histological grade and developed lymph node metastasis. Down-regulation of miR-338-3p was related with the recurrence $(P=0.005, O R=12.862,95 \% \mathrm{Cl}: 2.120 \sim 78.026)$ and death $(P=0.007$, $\mathrm{OR}=12.837,95 \% \mathrm{Cl}: 2.205 \sim 81.389$ ) of ovarian cancer patients, which was showed by binary Logistic regression analysis. Compared to other patients, the overall survival rate and progression free survival rate of patients with lower miR-338-3p and higher $\mathrm{MACCl}$ expression were obviously poorer $\left(\chi^{2}=16.955, P=7.219 \mathrm{E}-5 ; \chi^{2}=18.929, P=2.828 \mathrm{E}-5\right)$.

Conclusions Down-regulation of miR-338-3p and up-regulation of $M A C C 1$ gene were closely related with the poor prognosis of epithelial ovarian cancer patients, which could served as bio-markers of the progression and recurrence of ovarian cancer.
\end{abstract}

Key words: epithelial ovarian cancer, miR-338-3p, MACC1, recurrence, death

\section{Introduction}

Epithelial ovarian cancer (EOC) is commonly detected at a late stage, and mostly diagnosed with abdominal ascites and widespread intraperitoneal dissemination. Invasion and metastasis are the most 
important malignant biological characteristics of epithelial ovarian cancer, and its molecular mechanism is still not fully understood. Mutation and abnormal expression of microRNAs (miRNAs) are related to the occurrence and development of various human tumors. Most of miRNAs can inhibit or increase the expressions of many important tumor-related genes, and can act as oncogenes or tumor suppressor genes in the invasion and metastasis of malignant tumors [1].

The expression levels of microRNA-338-3p (miR-338-3p) in gastric cancer and colon cancer were significantly lower than those in normal paired tissues, down-regulation of miR-338-3p was related with the aggressive clinical characteristics (advanced clinical stage, lymph node or local metastasis) and poor prognosis of those malignant tumors[2-3]. MET transcriptional regulator MACC1 (MACC1) gene is abnormally up-expressed in many malignant tumor tissues such as colon cancer, gastric cancer, lung cancer and hepatocellular carcinoma, and is closely related to the invasion and metastasis of those malignant tumors, which can be used as a tumor marker to evaluate the prognosis and outcome of those malignant tumor patients [4-7].

Recently, several studies have shown that miR$338-3 p$ is down-expressed and MACC1 is up-regulated, and miR-338-3p can target the MACC1 gene in malignant tumor tissues such as gastric cancer, liver cancer, glioma cells and cervical cancer, which may be the molecular mechanism involved in the invasion and metastasis of those malignant tumors [8-11]. At present, the expressions of miR-338-3p and MACC1 in ovarian cancer have rarely been reported, the relationship between miR-338-3p and MACC1 genes in ovarian epithelial cancer tissues is still unclear.

Herein, real-time quantitative PCR was used to detect the expression of miR-338-3p and MACC1 genes in normal ovarian tissues, benign ovarian tumor tissues and ovarian epithelial carcinoma tissues, and their correlations was analyzed in this study. Furthermore, the relationships between their abnormal expressions and the clinical pathological parameters of ovarian cancer were explored, the clinical significance of their abnormal expressions and their relationships with the prognosis and recurrence of ovarian cancer patients were analyzed.

\section{Material and Methods}

\section{Tissue specimens and clinical data}

Fresh ovarian tissue specimens of 105 patients with complete follow-up data were collected from October 2012 to October 2015 in the First Affiliated Hospital of Zhengzhou University. All enrolled patients were initially diagnosed and treated for gynecological diseases except pregnancy, did not received medication or radiation therapy before surgery, and did not have other organ or system severe disorders or malignant neoplasm diseases. Patients who did not meet the above criteria were excluded. HE staining was independently performed by two pathologists to confirm the histological characteristics of all ovarian tissue specimens (shown in figure 1). There were 20 cases of normal ovarian tissues (collected from patients who underwent bilateral adnexectomy or/and hysterectomy for multiple fibroids or unilateral ovarian tumors without contralateral ovary suffered), 20 cases of benign ovarian epithelial tumor tissues (including 16 cases of serous cystadenoma and 4 cases of mucinous cystadenoma, collected from patients who underwent ovarian cystectomy or adnexectomy for benign ovarian tumors), and 65 cases of primary lesions of newly diagnosed EOC. There were no significant differences in the general data of age and BMI among normal, benign and cancer patients. The collection and use of all tissue samples in this study were informed to the patient or family members, and were approved by the Ethics Committee of the First Affiliated Hospital of Zhengzhou University.

EOC patients were 28 to 76 years old, with a median age of 50 years. Early stage patients (stage I-II) underwent comprehensive staging, and advanced stage patients (stages III-IV) underwent aggressive cytoreductive surgery (residual lesions less than 1 $\mathrm{cm}$ ). The clinical stage of EOC patients (FIGO 2014) was 8 cases in stage I, 4 cases in stage II, 48 cases in stage III, 5 cases in stage IV (including 2 cases of abdominal wall muscle metastases, 3 cases of retroperitoneal non-lymph node metastases). The histological grade of EOC patients was 38 cases at high grade and 27 cases at low grade. The histological classification was 52 cases of serous carcinoma and 13 cases of mucinous carcinoma. The retroperitoneal lymph node dissection reached the level of the inferior mesenteric artery. There were 32 cases with lymph node metastasis, including 26 cases with pelvic lymph node metastasis, 1 case with only para-aortic lymph node metastasis and 5 cases with both pelvic lymph node and para-aortic lymph node metastasis. Another 33 cases were free of lymph node metastasis. EOC patients received standard chemotherapy (Docetaxel plus Cisplatin or Carboplatin) after surgery for 3 to 6 cycles depending on the clinical stage. Patients developed drug resistance or recurrence after surgery were treated with second-line chemotherapy alone selective drugs or combination chemotherapy, including Docetaxel, Gemcitabine, Etoposide, Liposomal Doxorubicin and Oxaliplatin. 


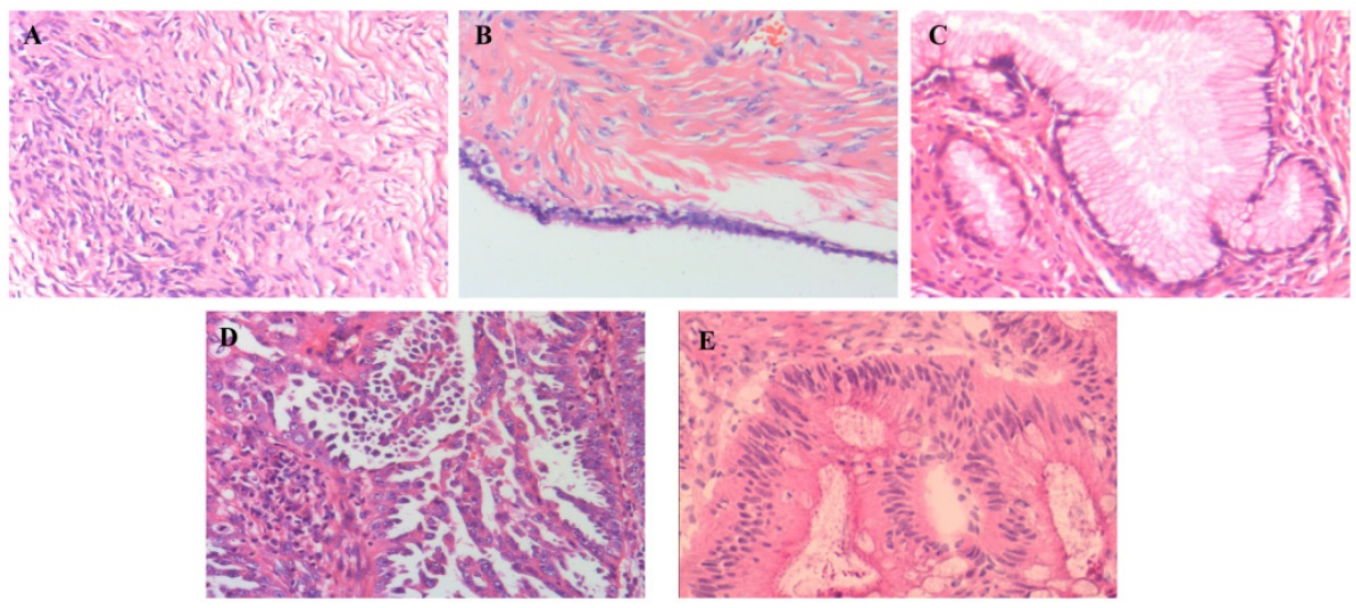

Figure 1. Microscopic pathological diagnosis of different ovarian tissue by HE staining $\times 100$. (Representative images were shown from 105 specimens of enrolled patients. A: normal ovarian tissue, B: ovarian serous cystadenoma tissue, C: ovarian mucinous cystadenoma tissue, D: ovarian serous cystadenocarcinoma tissue, E: ovarian mucinous cystadenocarcinoma tissue).

\section{Real time PCR}

Tissue total RNA was extracted by Trizol reagent (Invitrogen, Carlsbad, Calif., USA), quantified by spectrophotometry after purification. Reverse-synthesize cDNA template was synthesized from $2 \mu \mathrm{g}$ of total RNA for detection of miR-338-3p expression following the TaqMan MicroRNA Reverse Transcription kit instructions ( Applied Biosystems Co., Ltd. USA), with U6 as an normalization control. Another 2 $\mu \mathrm{g}$ of total RNA was reverse-transcribed into a cDNA template according to the protocol of RevertAid first strand cDNA synthesis kit (Fermentas, EU) for the detection of MACC1 gene expression, with $\beta$-actin as an internal reference. The real time PCR reaction was performed following the protocol of SYBR Green PCR kit (Dalian Takara, China) with Applied Biosystems 7500 Fast Real-Time PCR System (Thermo Fisher Scientific, USA). The primers used in PCR were synthesized by Shanghai Bioengineering Co., Ltd. The primer sequences are shown in Table 1 . The PCR reaction conditions were pre-denaturation at $95^{\circ} \mathrm{C}$ for $10 \mathrm{~min}$, denaturation at $95^{\circ} \mathrm{C}$ for $15 \mathrm{~s}$, and annealing at $60{ }^{\circ} \mathrm{C}$ for $60 \mathrm{~s}$ for 40 cycles. Each PCR experiment was performed for three times independently, and the relative expression value was expressed by $2^{-\Delta \Delta C t}$ method.

Table 1. Primer sequences for real time PCR

\begin{tabular}{lll}
\hline Item & Sequences $\left(\mathbf{5}^{\prime}\right.$ to $\left.\mathbf{3}^{\prime}\right)$ & $\begin{array}{l}\text { Product } \\
\text { length(bp) }\end{array}$ \\
\hline hsa-miR-338-3p & $\begin{array}{l}\text { Forward: AACCGGTCCAGCATCAGTGATT } \\
\text { Reverse: CAGTGCAGGGTCCGAGGT }\end{array}$ & 74 \\
U6 & $\begin{array}{l}\text { Forward: CTCGCTTCGGCAGCACA } \\
\text { Reverse: AACGCTTCACGAATTTGCGT }\end{array}$ & 70 \\
MACC1 & $\begin{array}{l}\text { Forward: CATTTTCGGTCAGGAAGAATTGC } \\
\text { Reverse: TGGAAGCATTATTACCACGAAGG }\end{array}$ & \\
\multirow{3}{*}{-actin } & $\begin{array}{l}\text { Forward: CATGTACGTTGCTATCCAGGC } \\
\text { Reverse: CTCCTTAATGTCACGCACGAT }\end{array}$ & 250 \\
\hline
\end{tabular}

\section{Statistical Analysis}

Average values were expressed as mean \pm standard deviation. Measurement data were analyzed by one-way ANOVA, non-parametric test, Bonferroni test, Independent sample T test, Spearman correlation test, binary Logistic regression analysis and KaplanMeier analysis using SPSS21.0 and GraphPad Prism 7 software package. Counting data were analyzed by Chi-square test or Fisher exact probability method. Difference was considered significant when $P$ value was less than 0.05 .

\section{Results}

\section{Expression of $\mathrm{miR-338-3p}$ and MACCl in different ovarian tissues}

In normal ovarian tissue, benign ovarian tumor tissue and EOC tissue, the relative expressions of miR-338-3p were $(0.821 \pm 0.008),(0.781 \pm 0.013)$ and $(0.331 \pm 0.038)$, the relative expressions of MACC1 were $(0.059 \pm 0.011),(0.315 \pm 0.034)$ and $(0.774 \pm 0.025)$. The expression of miR-338-3p in epithelial cancer tissues was significantly lower than that in normal ovarian tissue and benign ovarian tumor tissues $(\mathrm{F}=77.916$, $P=1.205 \mathrm{E}-18)$, but the expression of MACC1 was obviously higher in epithelial cancer tissues $(\mathrm{F}=$ 77.945, $P=1$.187E-18). Shown in figure 2 and 3.

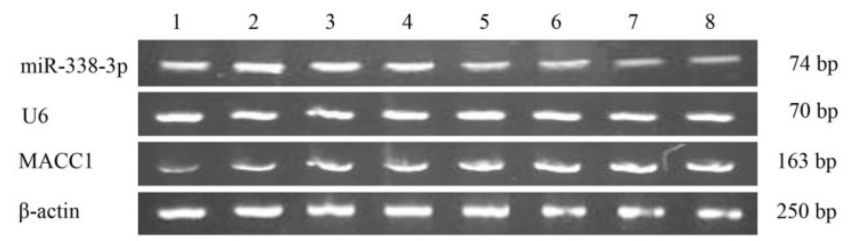

Figure 2. Expression of miR-338-3p and MACCl gene in different ovarian tissues explored by real time PCR. (Number 1-2 lanes: normal ovarian tissue, 3-4: benign epithelial ovarian tumor tissue, 5-8: EOC tissue). 

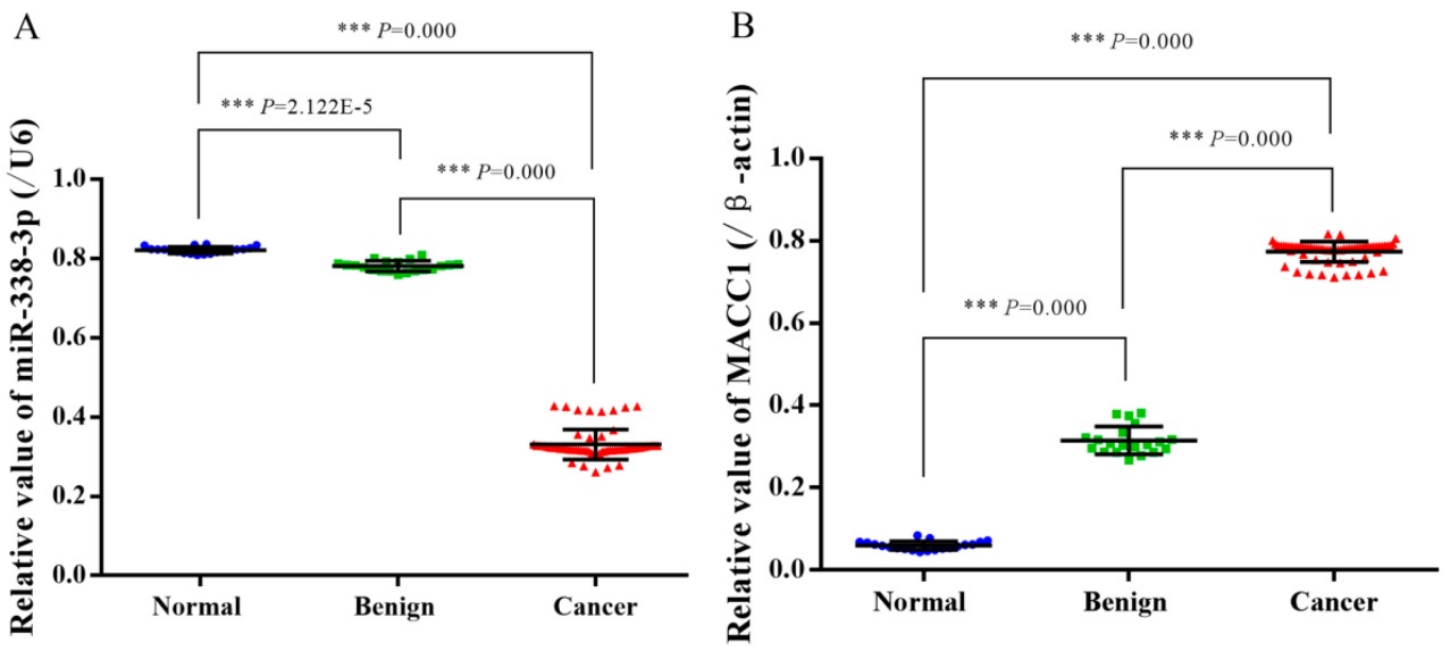

Figure 3. Expression of miR-338-3p and MACCI gene in different ovarian tissues shown by Scatter plot. (A: Relative values of miR-338-3p in different ovarian tissues, B: Relative values of MACCl gene in different ovarian tissues, ${ }^{* * *} P<0.001$ )

\section{The correlation between expressions of miR-338-3p and MACC1 in different ovarian tissues}

In 105 specimens different ovarian tissues, a significant negative correlation between miR-338-3p and MACC1 gene expression was detected by Spearman correlation test $(r=-0.968, P<0.0001)$, shown in figure 4 .

\section{The relationships between $\mathrm{miR}-338-3 p$ and MACCl expression and pathological parameters in EOC tissues}

The median relative expression levels of miR-338-3p and MACC1 in 65 cases of epithelial ovarian cancer were $(0.320 \pm 0.014)$ and $(0.782 \pm 0.033)$. According to the median expression, the expressions of miR-338-3p and MACC1 were divided into relatively high expression group (higher than or equal to the median expression) and relatively low expression group (lower than the median expression). In EOC tissues with more advanced clinical stage, higher histological grade and developed lymph node metastasis, the expression of miR-338-3p was obviously lower and the expression of MACC1 was significantly higher (Table 2).

\section{The high risk factors of relapse and death to the EOC patients}

In 65 EOC patients, the relations of miR-338-3p expression, MACC1 gene expression, age, clinical stage, histological grade, histological classification, ascites and lymph node metastasis with the recurrence and death of EOC were explored by binary Logistic regression analysis. The results indicated down-regulation of miR-338-3p $(P=0.005$, OR=12.862, 95\%CI: 2.120 78.026) and developed lymph node metastasis $(P=0.002, \mathrm{OR}=45.318,95 \% \mathrm{CI}: 4.010 \sim 512.120)$ were high risk factors of the recurrence in EOC patients. Moreover, down-regulation of miR-338-3p $(P=0.007, \quad O R=12.837, \quad 95 \% \mathrm{CI}: \quad 2.205 \sim 81.389) \quad$ and developed lymph node metastasis $(P=0.002$, OR= 45.381, 95\%CI: 3.945 521.975) also were high risk factors of the death in EOC patients. Multivariate regression analysis showed that none of the above factors were independent risk factors for the recurrence and death of EOC patients.

\section{The relations of $\mathrm{miR}-338-3 \mathrm{p}$ and $\mathrm{MACCl}$ gene expression with the overall survival and progression free survival of EOC patients analyzed by Kaplan-Meier analysis}

Up to February 2018, the follow-up time of 105 cases was 16 to 61 months, with a median follow-up of 38 months. At the end of the follow-up, 46 people survived and 19 died (Table 3). According to the median expression, the expressions of miR-338-3p and MACC1 were divided into relatively high expression group (higher than or equal to the median expression) and relatively low expression group (lower than the median expression) in EOC tissues. In 65 EOC patients, the overall survival rate of the EOC patients with low expression of miR-338-3p and high expression of MACC1 was significantly shorter than those patients with high miR-338-3p expression and high MACC1 expression, with high miR-338-3p expression and low MACC1 expression, and with low miR-338-3p expression and low MACC1 expression $\left(\chi^{2}=16.955, P=7.219 \mathrm{E}-5\right)$. Moreover, the progression free survival rate of the EOC patients with low expression of miR-338-3p and high expression of MACC1 also was obviously shortest in 65 EOC patients $\left(\chi^{2}=18.929, P=2.828 \mathrm{E}-5\right)$, which were shown in Figure 5 and 6. 
Table 2. The relationships between miR-338-3p and MACCl gene expressions in EOC tissues and clinicopathological parameters of $\operatorname{EOC}(n, \%)$

\begin{tabular}{|c|c|c|c|c|c|c|c|c|c|}
\hline \multirow[t]{2}{*}{ Item } & \multirow[t]{2}{*}{$\mathbf{n}$} & \multicolumn{2}{|c|}{ MACC1 } & \multirow[t]{2}{*}{$x^{2}$ value } & \multirow[t]{2}{*}{$P$ value } & \multicolumn{2}{|c|}{ miR-338-3p } & \multirow[t]{2}{*}{$\chi^{2}$ value } & \multirow[t]{2}{*}{$P$ value } \\
\hline & & High & Low & & & High & Low & & \\
\hline \multicolumn{10}{|l|}{ Age } \\
\hline$<50$ year & 31 & 14(45.16) & $17(54.84)$ & & & $17(54.84)$ & $14(45.16)$ & & \\
\hline$\geq 50$ year & 34 & $19(55.88)$ & $15(44.12)$ & 0.746 & 0.388 & $20(58.82)$ & 14(41.18) & 0.015 & 0.746 \\
\hline \multicolumn{10}{|c|}{ FIGO stage } \\
\hline I-II & 12 & $0(0.00)$ & $12(100.00)$ & & & $12(100.00)$ & $0(0.00)$ & & \\
\hline III-IV & 53 & $33(62.26)$ & $20(37.74)$ & & $5.506 \mathrm{E}-6^{* \star * \#}$ & $25(47.17)$ & $28(52.83)$ & & $0.001^{* \# \#}$ \\
\hline \multicolumn{10}{|c|}{ Histological grade } \\
\hline Low & 27 & $5(18.52)$ & $22(81.48)$ & & & $20(74.07)$ & $7(25.93)$ & & \\
\hline High & 38 & $28(73.68)$ & $10(26.32)$ & 19.219 & $1.384 \mathrm{E}-6^{* \star \star}$ & $17(44.74)$ & $21(55.26)$ & 5.540 & $0.019^{*}$ \\
\hline \multicolumn{10}{|c|}{ Histological classification } \\
\hline Serous & 52 & $29(56.60)$ & $23(43.40)$ & & & $28(53.85)$ & $24(46.15)$ & & \\
\hline Mucinous & 13 & $4(30.77)$ & $9(69.23)$ & 1.697 & 0.193 & $9(69.23)$ & $4(30.77)$ & 0.474 & 0.491 \\
\hline \multicolumn{10}{|l|}{ Ascites } \\
\hline No & 16 & $5(31.25)$ & $11(68.75)$ & & & $12(75.00)$ & $4(25.00)$ & & \\
\hline Yes & 49 & $28(57.14)$ & $21(42.86)$ & 3.235 & 0.072 & $25(51.02)$ & $24(48.98)$ & 1.935 & 0.164 \\
\hline \multicolumn{10}{|c|}{ Lymph node metastasis } \\
\hline No & 33 & 11(33.33) & $22(66.67)$ & & & $23(69.70)$ & $10(30.30)$ & & \\
\hline Yes & 32 & $22(68.75)$ & $10(31.25)$ & 8.153 & $0.004^{* *}$ & $14(43.75)$ & $18(56.25)$ & 4.461 & $0.035^{*}$ \\
\hline
\end{tabular}

${ }^{*} P<0.05,{ }^{* *} P<0.01,{ }^{* * *} P<0.001$, "Fisher exact probability method.

\section{Discussion}

Epithelial ovarian cancer is still one of the important diseases that threaten women's reproductive health. Most EOC patients are detected at advanced clinical stage, widespread invasion and metastasis in the pelvic and abdominal cavity is strongly responsible for the poor treatment and poor prognosis of EOC patients. Further research on the molecular mechanism of ovarian cancer invasion and metastasis is a serious problem that is urgently to be solved in the basic research and clinical treatment of ovarian cancer.

MicroRNAs, also called miRNAs, are endogenous, non-coding small RNAs that are highly evolutionarily conserved. There are more than a thousand miRNAs in the human genome. These miRNAs regulate the expressions of about one third of the protein-coding genes. They are the largest class of gene expression regulators and play important roles in a variety of physiological and pathological processes [12-13]. MiRNAs are closely related with the epithelial-mesenchymal transition, angiogenesis, tumor cell microenvironment and other malignant tumor invasion and metastasis processes, are also reported to participate in the invasion and metastasis of various malignant tumors including ovarian cancer, and can be used as a potential treatment target for malignant tumors [14-16].

It had been proved that miR-338-3p functioned as a tumor suppressor to inhibit cell proliferation and progression in numerous malignant tumors including gastric cancer, non-small-cell lung carcinoma, hepatocellular carcinoma, colorectal carcinoma, thyroid cancer and neuroblastoma [17-22]. In recent years, emerging researches have shown that miR-338-3p could inhibit the invasion and metastasis of malignant tumor cells by targeting different downstream carcinogenesis related genes in lung cancer, liver cancer, colorectal carcinoma, gastric cancer, renal cell carcinoma and nasopharyngeal carcinoma [23-28]. Up-regulation of MACC1 is proved to be a marker of the high aggressive ability of invasion and metastasis in numerous malignant tumor cells [29-33]. Our previous studies indicated that MACC1 mRNA and protein were over-expression in EOC tissues and cells, which is positively correlated with advanced clinical stage, and inhibition of MACC1 by RNA interference could suppress invasion and metastatic potential of ovarian carcinoma cells in vitro and in vivo[34-36].

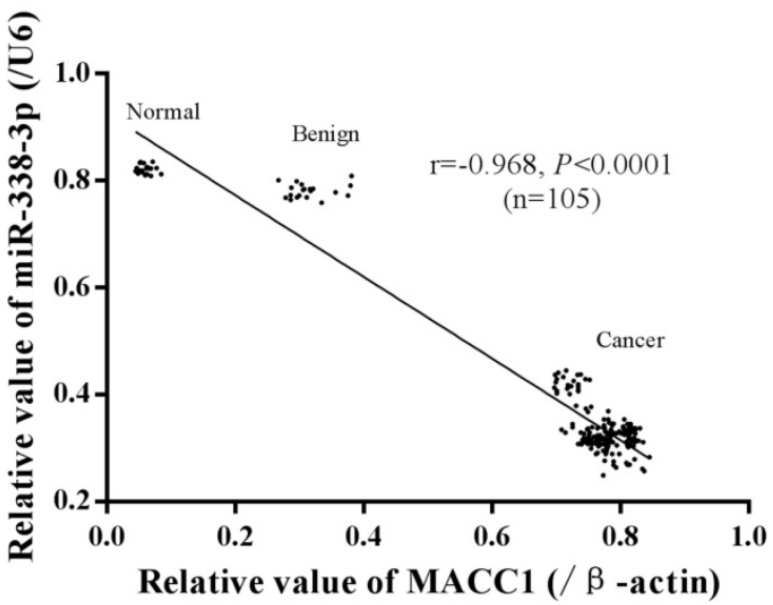

Figure 4. The correlation between miR-338-3p and MACCl gene expression in different ovarian tissues. 


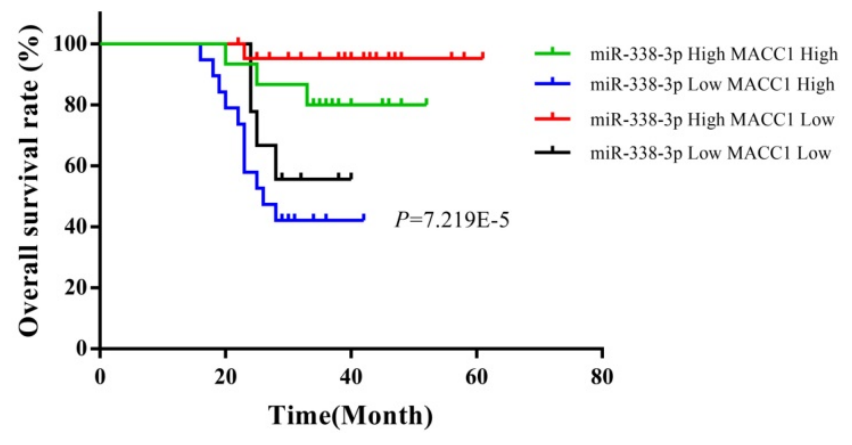

Figure 5. Relationships between miR-338-3p and MACCl expression and the overall survival of EOC patients.

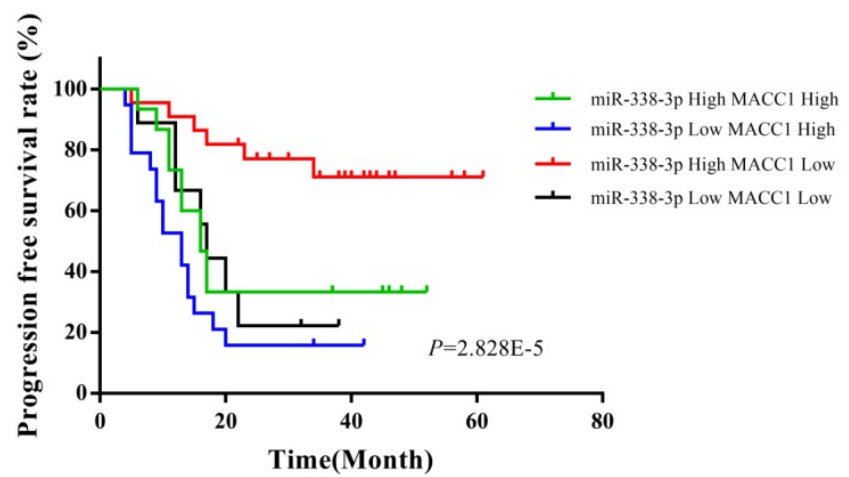

Figure 6. Relationships between miR-338-3p and $\mathrm{MACCl}$ expression and the progression free survival of EOC patients.

Table 3. The status of $65 \mathrm{EOC}$ patients at the end of follow-up

\begin{tabular}{llllll}
\hline FIGO stage & $\mathbf{n}$ & Stable & \multicolumn{2}{c}{ Recurrence } & Death \\
\cline { 5 - 6 } & & & 7 2 times & $\mathbf{2}$ times & \\
\hline I & 8 & 7 & 1 & 0 & 0 \\
II & 4 & 3 & 1 & 0 & 0 \\
III & 48 & 16 & 9 & 9 & 14 \\
IV & 5 & 0 & 0 & 0 & 5 \\
Total & 65 & 26 & 11 & 9 & 19 \\
\hline
\end{tabular}

In present study, real time-PCR was used to detect the expression of miR-338-3p and MACC1 gene in normal ovarian tissues, benign epithelial ovarian tumor tissues and EOC tissue. Compared with normal ovarian tissue and benign epithelial ovarian tumors, the expression of miR-338-3p was significantly decreased and the expression of MACC1 gene was significantly increased in EOC tissues, which suggested that abnormal expressions of miR-338-3p and MACC1 might be involved in the occurrence and development of ovarian cancer. Advanced clinical stage and developed local or lymph node metastasis means the aggressive ability of malignant tumor cells to invade and metastasize. For further analysis of our present research data, we had obtained some meaningful results. Down-regulation of miR-338-3p and up-regulation of MACC1 were positively correlated with advanced clinical stage, high histological grade and developed lymph node metastasis. Low expression of miR-338-3p and developed lymph node metastasis were associated with the recurrence and death of EOC patients. Overall survival and progression free survival were significantly shorter in EOC patients with low expression of miR-338-3p and high expression of MACC1.These results indicated that low expression of miR-338-3p and high expression of MACC1 might be closely associated with and served as bio-markers of the progression and recurrence of EOC.

Generally speaking, miRNAs participate in various physiological and pathological processes mainly by targeting the expression of downstream related genes or the activity of downstream signaling pathway. During the development of malignant tumors, most miRNAs play a regulatory role in oncogenes or tumor suppressor genes, and even the same miRNA plays an opposite regulatory role in different cancer tissues [37]. In ovarian cancer cells, rare report about the targeting and regulation of MACC1 gene by miR-338-3p was found. Our search revealed that MACC1 was a downstream potential target gene of miR-338-3p using TargetScan Human 7.1 and miRanda online database. Some other studies had confirmed that miR-338-3p could target and regulate the MACC1 gene to control the proliferation, invasion and metastasis of malignant tumors [8-11]. There was a significant negative correlation between the expression of miR-338-3p and MACC1 in different ovarian tissues, and EOC patients with low expression of miR-338-3p and high expression of MACC1 had the worst survival, which were shown by our present results. Due to these data, we speculate that miR-338-3p might target and regulate MACC1 in the carcinogenosis of ovarian cancer, at least partly, which should be verified by further molecular experiments.

However, the expression profiles and prognostic value of miR-338-3p and MACC1 in normal ovarian and ovarian cancer tissues were rarely reported. It was indicated that significantly downregulated expressions of miR-338-3p was detected by real-time quantitative RT-PCR in 54 specimens ovarian epithelial carcinoma tissues, which was negatively related to advanced FIGO stage, high histological grading and lymph node metastasis [38]. Moreover, miR-338-3p expression was shown to be downregulated in ovarian cancer tissues and cell lines, and the regulation of miR-338-3p to downstream target gene may play important roles in ovarian cancer cell proliferation and metabolism [39]. Up-regulation of MACC1 has been proved to indicate poor progression in many malignant tumors, including ovarian cancer. In one report including 207 specimens EOC tissue 
samples, significant overexpressions of MACC1 were detected by immunohistochemistry, which were significantly associated with high tumor/lymph node/metastasis grade, non-primary site implantation, advanced clinical stage and shorter overall survival time [40]. Overexpressions of MACC1 were also explored in EOC tissue and serum samples in another independent research, which indicated MACC1 was closely related with the lymph node metastasis, advanced clinical stage, high histological grade and poor prognosis of EOC patients [41]. In ovarian cancer, advanced FIGO stage, high histological grade, lymph node metastasis and distance implantation usually indicates poor prognosis and short survival time. Combined with the previous reports from other malignant tumors and the recent studies in EOC, the present study explored a preliminary expression profiles and prognostic value of miR-338-3p and MACC1 for ovarian cancer. Due to the limited number of patients observed, the clinical significance of our results is still need to be verified by further researches. Therefore, larger numbers, more comprehensive clinical records, more closely follow-up, and multicenter cooperation cohort researches need to be developed in future to accurately elucidate the expression and prognostic value of miR-338-3p and MACC1 in ovarian cancer.

\section{Conclusions}

In summary, this study showed low expression of miR-338-3p and high expression of MACC1 were detected in EOC tissues, which might be closely associated with and served as bio-markers of the progression and recurrence of EOC. In order to confirm our speculations about miR-338-3p targets and regulates MACC1 in ovarian cancer, further molecular studies should be developed, which can help to comprehensively elucidate the molecular mechanism of invasion and metastasis of ovarian cancer, and can provide a potential molecular target or a new direction for ovarian cancer precise treatment.

\section{Abbreviations}

miR-338-3p: microRNA-338-3p; MACC1: MET transcriptional regulator MACC1; EOC: epithelial ovarian cancer.

\section{Acknowledgments}

We thank Professor Junming Yue from the University of Tennessee Health Science Center for reviewing the revision manuscript, and thank the Clinical Medicine Key Disciplines Laboratory of Henan Province for assistance with experiments. Our work was supported by the Key Scientific Research
Projects of Advanced Education Institutions of Department of Education of Henan Province (19A320006), in part by the Health and Family Planning Technology Overseas Training Project of Henan Province (2018028), and in part by the Youth Innovation Fund Project of the First Affiliated Hospital of Zhengzhou University (201418).

\section{Competing Interests}

The authors have declared that no competing interest exists.

\section{References}

1. Iorio MV, Croce CM. microRNA involvement in human cancer. Carcinogenesis. 2012;33:1126-33.

2. Liu S, Suo J, Wang C, Sun X, Wang D, He L, et al. Downregulation of tissue miR-338-3p predicts unfavorable prognosis of gastric cancer. Cancer Biomark. 2017;21:117-22.

3. Sun K, Su G, Deng H, Dong J, Lei S, Li G. Relationship between miRNA-338-3p expression and progression and prognosis of human colorectal carcinoma. Chin Med J (Engl). 2014;127:1884-90.

4. Shirahata A, Shinmura K, Kitamura Y, Sakuraba K, Yokomizo K, Goto T, et al. MACC1 as a marker for advanced colorectal carcinoma. Anticancer Res. 2010;30:2689-92.

5. Shirahata A, Sakata M, Kitamura Y, Sakuraba K, Yokomizo K, Goto T, et al. MACC 1 as a marker for peritoneal-disseminated gastric carcinoma. Anticancer Res. 2010; 30:3441-4.

6. Shimokawa H, Uramoto H, Onitsuka T, Chundong G, Hanagiri T, Oyama T, et al. Overexpression of MACC1 mRNA in lung adenocarcinoma is associated with postoperative recurrence. J Thorac Cardiovasc Surg. 2011;141:895-8.

7. Shirahata A, Fan W, Sakuraba K, Yokomizo K, Goto T, Mizukami H, et al. MACC 1 as a marker for vascular invasive hepatocellular carcinoma. Anticancer Res. 2011;31:777-80.

8. Huang N, Wu Z, Lin L, Zhou M, Wang L, Ma H, et al. MiR-338-3p inhibits epithelial-mesenchymal transition in gastric cancer cells by targeting ZEB2 and MACC1/Met/Akt signaling. Oncotarget. 2015;6:15222-34.

9. Zhang T, Liu W, Zeng XC, Jiang N, Fu BS, Guo Y, et al. Down-regulation of microRNA-338-3p promoted angiogenesis in hepatocellular carcinoma. Biomed Pharmacother. 2016;84:583-91.

10. Shang C, Hong Y, Guo Y, Xue YX. Mir-338-3p inhibits malignant biological behaviors of glioma cells by targeting MACC1 gene. Med Sci Monit. 2016;22:710-6.

11. Hua FF, Liu SS, Zhu LH, Wang YH, Liang X, Ma N, et al, MiRNA-338-3p regulates cervical cancer cells proliferation by targeting MACC1 through MAPK signaling pathway. Eur Rev Med Pharmacol Sci. 2017;21:5342-52.

12. Lewis BP, Burge CB, Bartel DP. Conserved seed pairing, often flanked by adenosines, indicates that thousands of human genes are microRNA targets. Cell. 2005;120:15-20.

13. Hayes J, Peruzzi PP, Lawler S. MicroRNAs in cancer: biomarkers, functions and therapy. Trends Mol Med, 2014,20(8):460-9.

14. Toden S, Okugawa Y, Jascur T, Wodarz D, Komarova NL, Buhrmann C, et al. Curcumin mediates chemosensitization to 5-fluorouracil through miRNA-induced suppression of epithelial-to-mesenchymal transition in chemoresistant colorectal cancer. Carcinogenesis. 2015;36:355-67.

15. Shen G, Li X, Jia YF, Piazza GA, Xi Y. Hypoxia-regulated microRNAs in human cancer. Acta Pharmacol Sin. 2013;34:336-41.

16. Slaby $\mathrm{O}$, Laga $\mathrm{R}$, Sedlacek $\mathrm{O}$. Therapeutic targeting of non-coding RNAs in cancer. Biochem J. 2017;474:4219-51

17. Sun F, Yu M, Yu J, Liu Z, Zhou X, Liu Y, et al. miR-338-3p functions as a tumor suppressor in gastric cancer by targeting PTP1B. Cell Death Dis. 2018;9:522.

18. Sun J, Feng X, Gao S, Xiao Z. microRNA-338-3p functions as a tumor suppressor in human non-small-cell lung carcinoma and targets Ras-related protein 14. Mol Med Rep. 2015;11:1400-6.

19. Wang G, Sun Y, He Y, Ji C, Hu B, Sun Y. MicroRNA-338-3p inhibits cell proliferation in hepatocellular carcinoma by target forkhead box P4 (FOXP4). Int J Clin Exp Pathol. 2015;8:337-44.

20. Sun K, Deng HJ, Lei ST, Dong JQ, Li GX. miRNA-338-3p suppresses cell growth of human colorectal carcinoma by targeting smoothened. World J Gastroenterol. 2013;19:2197-207.

21. Sui GQ, Fei D, Guo F, Zhen X, Luo Q, Yin S, et al. MicroRNA-338-3p inhibits thyroid cancer progression through targeting AKT3. Am J Cancer Res. 2017;7:1177-87.

22. Chen X, Pan M, Han L, Lu H, Hao X, Dong Q. miR-338-3p suppresses neuroblastoma proliferation, invasion and migration through targeting PREX2a. FEBS Lett. 2013;587:3729-37.

23. Li Y, Chen P, Zu L, Liu B, Wang M, Zhou Q. MicroRNA-338-3p suppresses metastasis of lung cancer cells by targeting the EMT regulator Sox4. Am J Cancer Res. 2016;6:127-40. 
24. Huang $\mathrm{XH}$, Chen JS, Wang $\mathrm{Q}$, Chen $\mathrm{XL}$, Wen $\mathrm{L}$, Chen LZ, et al. miR-338-3p suppresses invasion of liver cancer cell by targeting smoothened. J Pathol. 2011;225:463-72.

25. Xue Q, Sun K, Deng HJ, Lei ST, Dong JQ, Li GX. MicroRNA-338-3p inhibits colorectal carcinoma cell invasion and migration by targeting smoothened. Jpn J Clin Oncol. 2014;44:13-21.

26. Chen JT, Yao KH, Hua L, Zhang LP, Wang CY, Zhang JJ. MiR-338-3p inhibits the proliferation and migration of gastric cancer cells by targeting ADAM17. Int J Clin Exp Pathol. 2015;8:10922-8.

27. Tong Z, Meng X, Wang J, Wang L. MicroRNA-338-3p targets SOX4 and inhibits cell proliferation and invasion of renal cell carcinoma. Exp Ther Med. 2017 Nov;14(5):5200-5206.

28. Shan Y, Li X, You B, Shi S, Zhang Q, You Y. MicroRNA-338 inhibits migration and proliferation by targeting hypoxia-induced factor $1 \alpha$ in nasopharyngeal carcinoma. Oncol Rep. 2015;34:1943-52.

29. Stein U, Walther W, Arlt F, Schwabe H, Smith J, Fichtner I, et al. MACC1, a newly identified key regulator of HGF-MET signaling, predicts colon cancer metastasis. Nat Med. 2009;15:59-67.

30. Wang L, Wu Y, Lin L, Liu P, Huang H, Liao W, et al. Metastasis-associated in colon cancer-1 upregulation predicts a poor prognosis of gastric cancer, and promotes tumor cell proliferation and invasion. Int J Cancer. 2013;133:1419-30

31. Sheng XJ, Li Z, Sun M, Wang ZH, Zhou DM, Li JQ, et al. MACC1 induces metastasis in ovarian carcinoma by upregulating hepatocyte growth factor receptor c-MET. Oncol Lett. 2014;8:891-7.

32. Wang $Y$, Hong Q, Wang J, Fang $Y$, Hu C. Downregulated expression of metastasis associated in colon cancer 1 (MACC1) reduces gallbladder cancer cell proliferation and invasion. Tumour Biol. 2014;35:3771-8.

33. Ding Y, Li X, Hong D, Jiang L, He Y, Fang H. Silence of MACC1 decreases cell migration and invasion in human malignant melanoma through inhibiting the EMT. Biosci Trends. 2016;10:258-64.

34. Zhang RT, Shi HR, Huang HL, Chen ZM, Liu HN, Yuan ZF. Expressions of MACC1, HGF, and C-met protein in epithelial ovarian cancer and their significance. Nan Fang Yi Ke Da Xue Xue Bao. 2011;31:1551-5.

35. Zhang RT, Ren F, Shi HR. Expression of metastasis-associated in colon cancer-1 in different stages of epithelial ovarian cancer. Zhongguo Yi Xue Ke Xue Yuan Xue Bao. 2014;36:47-51.

36. Zhang RT, Shi HR, Chen ZM, Wu QH, Ren F, Huang HL. Effects of metastasis-associated in colon cancer 1 inhibition by small hairpin RNA on ovarian carcinoma OVCAR-3 cells. J Exp Clin Cancer Res. 2011;30:83.

37. Zamore PD, Haley B. Ribo-gnome: the big world of small RNAs. Science. 2005; 309:1519-24.

38. Wen C, Liu X, Ma H, Zhang W, Li H. miR-338-3p suppresses tumor growth of ovarian epithelial carcinoma by targeting Runx2. Int J Oncol. 2015; 46: 2277-85.

39. Zhang Y, Shi B, Chen J, Hu L, Zhao C. MiR-338-3p targets pyruvate kinase M2 and affects cell proliferation and metabolism of ovarian cancer. Am J Transl Res. 2016; 8:3266-73.

40. Yu L, Zhu B, Wu S, Zhou L, Song W, Gong X, et al. Evaluation of the correlation of vasculogenic mimicry, ALDH1, KiSS-1, and MACC1 in the prediction of metastasis and prognosis in ovarian carcinoma. Diagn Pathol. 2017;12:23.

41. Li H, Zhang $\mathrm{H}$, Zhao S, Shi $\mathrm{Y}$, Yao J, Zhang $\mathrm{Y}$, et al. Overexpression of MACC1 and the association with hepatocyte growth factor/c-Met in epithelial ovarian cancer. Oncol Lett. 2015;9:1989-96. 